\title{
Existenz von Quanten-Yang-Mills-Theorien mit Massenlücke
}

\author{
Elmar Vogt
}

Um Enttäuschungen vorzubeugen: Auch nach Lektüre dieses Artikels wird es ausser denen, die eh schon Bescheid (und damit deutlich mehr als ich) wissen, nicht klar sein, was genau zu tun ist, um folgendes Problem zu lösen:

Beweise, dass für jede kompakte einfache Liegruppe eine Quanten-Yang-MillsTheorie auf der 4-dimensionalen Raum-Zeit existiert, die eine positive Massenlücke besitzt.

Eine (klassische) Yang-Mills-Theorie ist eine Eichfeldtheorie. Unter einer Eichfeldtheorie über einer Raum-Zeit, die wir uns allgemein $d$-dimensional denken, stellt man sich am einfachsten eine Familie endlich dimensionaler Vektorräume $V_{x}$ vor, indiziert durch die Punkte $x \in \mathbb{R}^{d}$ der Raum-Zeit, die jeweils die ,Zustände“ im Punkt $x$ beschreiben. Das Fehlen unmittelbarer Wirkungen zwischen verschiedenen Punkten $x$ und $y$ von $\mathbb{R}^{d}$ verhindert es, Vektoren in $V_{x}$ und $V_{y}$ zu vergleichen, selbst wenn $V_{x}$ und $V_{y}$ isomorph sind.

Um dennoch Ableitungen von Feldern definieren zu können, also von Funktionen, die den Punkten $x \in \mathbb{R}^{d}$ Vektoren aus $V_{x}$ zuordnen, führt man so genannte Eichfelder ein. Wir machen dies exemplarisch für den Fall, dass alle $V_{x}$ als euklidische Vektorräume isomorph zu $\mathbb{R}^{2}$ sind. Es ist dann günstig, die $V_{x}$ mit $\mathbb{C}$ und die Drehgruppe des $\mathbb{R}^{2}$ mit $S^{1}=\{z \in \mathbb{C}:\|z\|=1\}$ zu identifizieren. $S^{1}$ operiert durch Multiplikation auf $\mathbb{C}$, und dies entspricht den Drehungen des $\mathbb{R}^{2}$.

In dieser Situation ordnet ein Eichfeld jedem (differenzierbaren) Weg $w:[0,1] \rightarrow \mathbb{R}^{d}$ des $\mathbb{R}^{d}$ ein Element $g_{w} \in S^{1}$ zu. Dies interpretieren wir in der Weise, dass für $x=w(0)$ und $y=w(1)$ der Vektor $v \in V_{x}$ dem Vektor $g_{w} \cdot v \in V_{y}$ entspricht. Sind dabei $w_{1}, w_{2}$ Wege, die zusammengesetzt werden können zu $w=w_{1} * w_{2}$, so soll $g_{w}=g_{w_{2}} \cdot g_{w_{1}}$ sein. Infinitesimal heisst das, dass wir jedem Punkt $x$ des $\mathbb{R}^{d}$ und Tangentialvektor $v$ des $\mathbb{R}^{d}$ im Punkt $x$ einen Tangentialvektor

$$
\omega_{x}(v)
$$

an $S^{1}$ im Punkt 1 zuordnen. Um das einzusehen, beachte, dass wir den Tangentialraum $T_{1} S^{1}$ an $S^{1}$ im Punkt 1 mit $i \cdot \mathbb{R}$ identifizieren können. 


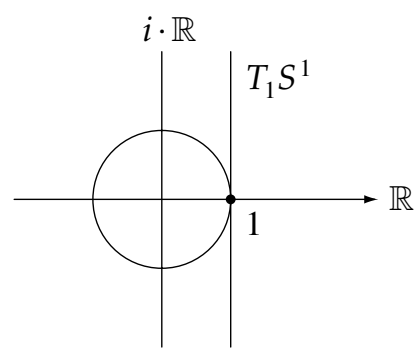

Ist dann $w:[0,1] \rightarrow \mathbb{R}^{d}$ ein differenzierbarer Weg von $x=w(0)$ nach $y=w(1)$, so können wir die Zuordnung $(x, v) \longmapsto \omega_{x}(v) \in i \cdot \mathbb{R}$ nutzen, um eine Differentialgleichung für Funktionen $f:[0,1] \rightarrow i \cdot \mathbb{R}$ aufzustellen:

$$
f^{\prime}(t)=\omega_{w(t)}\left(\frac{d w}{d t}(t)\right) .
$$

Ist $f$ die Lösung dieser Gleichung mit $f(0)=0$, so erhalten wir $g_{w}$ als $g_{w}=e^{f(1)}$.

Etwas salopper ausgedrückt ermöglicht es das Eichfeld, Basen des Vektorraums über dem Anfangspunkt eines Weges entlang dieses Weges in Basen der Vektorräume über Punkten des Weges zu verschieben und damit Vektoren über verschiedenen Punkten zu vergleichen.

Vergessen wir den Faktor $i$ in $i \cdot \mathbb{R}$, so ist die Zuordnung $(x, v) \longmapsto \omega_{x}(v)$ nichts anderes als eine 1 -Form auf $\mathbb{R}^{d}$, wie wir sie aus der Analysisvorlesung kennen. Allgemein hat eine solche die Form

$$
\omega=\sum_{i=1}^{d} a_{i} d x^{i} .
$$

Die $a_{i}$ sind dabei differenzierbare Funktionen auf $\mathbb{R}^{d}$ und $\omega_{x}(v)=\sum_{i=1}^{d} a_{i}(x) v^{i}$, wenn $v=\left(v^{1}, \ldots, v^{d}\right)$.

Nun kann es bei einem Eichfeld $\omega$ durchaus vorkommen, dass für einen geschlossenen Weg $w$, d.h. einen Weg $w$ mit $w(0)=w(1)$, das $g_{w}$ von der Identität verschieden ist, selbst wenn $w$ sehr kurz ist. Gemessen wird diese Tatsache dadurch, dass die 2-Form

$$
d \omega=\sum_{i, j} \frac{\partial a_{i}}{\partial x^{j}} d x^{j} \wedge d x^{i}
$$

von 0 verschieden sein kann. Diese 2-Form heisst für Mathematiker die Krümmung, für Physiker die Feldstärke des Eichfelds $\omega$. Warum?

Dazu betrachten wir das elektrische bzw. magnetische Feld $E=\left(E_{1}, E_{2}, E_{3}\right)$ bzw. $B=$ $\left(B_{1}, B_{2}, B_{3}\right)$. Diese gehorchen den Maxwellschen Gleichungen, die im Vakuum die Form

$$
\begin{aligned}
& \operatorname{rot} E+\frac{\partial}{\partial t} B=0, \quad \operatorname{div} B=0, \\
& \operatorname{rot} B-\frac{\partial}{\partial t} E=0, \quad \operatorname{div} E=0
\end{aligned}
$$


haben. Der Trick besteht nun darin, $E$ und $B$ als Felder in der 4-dimensionalen RaumZeit mit Koordinaten $x_{0}=$ Zeit, $\left(x_{1}, x_{2}, x_{3}\right)=$ Raum (Lichtgeschwindigkeit wird gleich 1 gesetzt) zu betrachten und zu einer 2 -Form im $\mathbb{R}^{4}$ zusammenzufassen:

$$
F=-\sum_{i=1}^{3} E_{i} d x^{0} \wedge d x^{i}+B_{1} d x^{2} \wedge d x^{3}+B_{2} d x^{3} \wedge d x^{1}+B_{3} d x^{1} \wedge d x^{2}
$$

Berechnen Sie jetzt zur Übung die 3-Form $d F$. Zu Ihrem Erstaunen werden Sie feststellen, dass die beiden ersten Maxwellgleichungen (1) gerade den Sachverhalt $d F=0$ ausdrücken. Aus der Analysis wissen wir dann, dass es eine 1-Form $\omega$ auf $\mathbb{R}^{4}$ gibt mit $d \omega=F$. Das $\omega$, das wissen wir, lässt sich als Eichfeld auffassen und $d \omega=F$ beschreibt das elektromagnetische Feld. Daher die Bezeichnung Feldstärke für $d \omega$.

Die letzten beiden Maxwellgleichungen haben auch eine geometrische Bedeutung, die sich auf die Minkowski-Metrik auf der Raum-Zeit bezieht. Mit Hilfe dieser Metrik lässt sich jeder $p$-Form $\nu$ auf der Raum-Zeit eine $(4-p)$-Form $* \nu$ zuordnen. Die MinkowskiMetrik (eigentlich eine Pseudometrik) definiert für Elemente $x, y$ aus $\mathbb{R}^{4}$ deren Skalarprodukt $\langle x, y\rangle$ durch $\langle x, y\rangle=x^{0} y^{0}-\sum_{i=1}^{3} x^{i} y^{i}$. Bezüglich dieser Metrik ist

$$
\begin{array}{ll}
* d x^{0} \wedge d x^{1}=d x^{2} \wedge d x^{3}, & * d x^{0} \wedge d x^{2}=d x^{3} \wedge d x^{1}, \\
* d x^{0} \wedge d x^{3}=d x^{1} \wedge d x^{2}, & * d x^{1} \wedge d x^{2}=d x^{3} \wedge d x^{0}, \\
* d x^{1} \wedge d x^{3}=d x^{0} \wedge d x^{2}, & * d x^{2} \wedge d x^{3}=d x^{1} \wedge d x^{0} .
\end{array}
$$

Damit kennen wir $* \nu$ für jede 2-Form $\nu$, und die beiden letzten Maxwellgleichungen (2) erhalten nun die Form

$$
d(* F)=0, \quad \text { bzw. } \quad d(* d \omega)=0 .
$$

Da $d(d \omega)=0$ für jedes $\omega$ gilt, lassen sich also die Maxwellgleichungen knapp in die Form

$$
d(* d \omega)=0
$$

bringen, wobei $\omega$ eine 1-Form ist und $d \omega$ das elektromagnetische Feld beschreibt; $\omega$ heisst auch das elektromagnetische Potential.

Nun wird gnadenlos verallgemeinert. Anstelle von $S^{1}$ nehmen wir $G=S U(n)$, die Gruppe der unitären komplexen $(n \times n)$-Matrizen mit Determinante 1 . Wer weiss, was das ist, darf auch gleich eine beliebige kompakte einfache Liegruppe $G$ nehmen. $S U(n)$ ist eine solche. Ein Eichfeld ist wieder eine Form, die einem Tangentialvektor $(x, v)$ im Punkt $x$ des $\mathbb{R}^{d}$ einen Tangentialvektor $\omega_{x}(v)$ in $G$ im neutralen Element $\operatorname{id}_{G}$ zuordnet. Operiert $G$ auf $V_{x}$ (z.B. operiert $S U(n)$ auf $\left.\mathbb{C}^{n}\right)$, so beschreibt die ,1-Form $\omega$ mit Werten im Tangentialraum g von $\operatorname{id}_{G}$ “ wieder den Transport von Basen entlang Wegen. Die Krümmung $F$ ist eine 2-Form mit Werten in $\mathfrak{g}$, nur lässt sie sich jetzt nicht einfach durch die Ableitung von Differentialformen beschreiben. Im Fall $G=S U(n)$ ist $\mathfrak{g}=$ : $s u(n)$ die additive Gruppe der Matrizen $A$ mit $A+\bar{A}^{t}=0$. Sind $A, B \in s u(n)$, so ist $[A, B]=A B-B A$ wieder aus $s u(n)$. Sind $v, w$ Tangentialvektoren des $\mathbb{R}^{d}$ in demselben Punkt, so haben wir nun

$$
F(v, w)=d \omega(v, w)+\frac{1}{2}[\omega(v), \omega(w)] .
$$


Das Analogon zu den ersten beiden Maxwellgleichungen ist hier $D F=0$, wobei $D$ die Ableitung mit Hilfe des Zusammenhangs $\omega$ ist, die so genannte kovariante Ableitung. Wie im Falle der Maxwellgleichungen ist diese Gleichung immer erfüllt. Dies ist die so genannte Bianchi-Identität.

Das Analogon der zweiten Maxwellgleichungen heisst die Yang-Mills-Gleichung. Im Vakuum lautet sie

$$
D(* F)=0 .
$$

Ein Yang-Mills-Feld ist ein Eichfeld $\omega$, dessen Krümmung (Feldstärke) F die Yang-MillsGleichung erfüllt. Wegen des zweiten Summanden in $F$ ist dies eine nicht-lineare Gleichung für $\omega$. Physikalisch bedeutet dies, dass Yang-Mills-Felder für $G=S U(n), n \geq 2$, mit sich selbst in Wechselwirkung stehen. Das macht deren Dynamik kompliziert. Man erhält keine freien Felder.

Eichfeldtheorien sind physikalische Theorien, die durch ein oder mehrere Felder beschrieben werden, die an Eichfelder gekoppelt sind. Dabei soll die Theorie unabhängig von Eichungen sein. Das heisst, dass egal wie an verschiedenen Punkten $x$ die Basis von $V_{x}$ gewählt wird, die Theorie dieselben Lösungen liefert. Aus dem Schulunterricht kennen wir das nur global: Ändern wir unser gesamtes Bezugssystem durch Verschieben des Nullpunkts, Drehen der Koordinaten oder Addition einer überall gleichen Geschwindigkeit, so bleiben die Feldgleichungen unverändert. Bei einer Eichfeldtheorie darf man die Basis von Punkt zu Punkt ändern. Z.B. bleiben die Maxwellgleichungen unverändert, wenn wir $\omega$ durch $\omega+d\left(e^{i \cdot f}\right)$ ersetzen, wobei $f$ eine beliebige Funktion ist. Dies hat zur Konsequenz, dass unsere Eichfelder stets masselos sind, jedenfalls im klassischen Bereich. Reine Yang-Mills-Theorien sind Theorien, in denen nur das Eichfeld auftritt, keine weiteren Felder.

So viel zu den klassischen Feldtheorien. Bevor wir uns Gedanken zum Quantisieren von Feldtheorien machen, ist es nützlich, sich an die Grundprinzipien der Quantenmechanik zu erinnern.

In der klassischen Mechanik sind Zustände Punkte des Phasenraums, Observable (z.B. Ort, Energie oder Impuls) Funktionen auf dem Phasenraum. Die Zustände eines quantenmechanischen Systems sind 1-dimensionale Unterräume eines komplexen Hilbertraums $\mathscr{H}$, also Einheitsvektoren von $\mathcal{H}$, die nur bis auf einen Phasenfaktor, d.h. bis auf Multiplikation mit einer komplexen Zahl vom Betrag 1, bestimmt sind. Dies erlaubt die wahrscheinlichkeitstheoretische Interpretation in der Quantenmechanik. Gegeben sei ein System im Zustand $\varphi$. Die Wahrscheinlichkeit, dass es sich im Zustand $\psi$ befindet, ist dann $\langle\varphi, \psi\rangle \cdot\langle\psi, \varphi\rangle$, wobei $\langle$,$\rangle das innere Produkt des Hilbertraums \mathscr{H}$ ist. Das ist sinnvoll, denn es ist $0 \leq\langle\varphi, \psi\rangle \cdot\langle\psi, \varphi\rangle \leq 1$.

Observable sind selbstadjungierte Operatoren von $\mathscr{H}$. Messungen liefern Eigenwerte des Operators. Der Energie- oder Hamiltonoperator $H$ ist der infinitesimale Erzeuger der zeitlichen Entwicklung des Systems: Der Zustand $\psi$ im Zeitpunkt 0 geht zum Zeitpunkt $t$ in den Zustand

$$
\psi(t)=e^{-i t H / \hbar}(\psi)
$$

über. Analog beschreiben die Impulsoperatoren infinitesimal die Raumtranslationen und die Drehimpulsoperatoren die Raumdrehungen. 
Ein gängiges Modell für $\mathscr{H}$ sind die $L^{2}$-integrierbaren Funktionen auf $\mathbb{R}^{3}$, wenn man ein Teilchen beschreibt. Zustände sind also Felder, und ist $H$ der Hamiltonoperator, so gehorcht die zeitliche Entwicklung $\psi(t)$ eines Feldes $\psi=\psi(0)$ wegen (3) der Schrödingergleichung

$$
i \hbar \frac{d \psi(t)}{d t}=H(\psi(t)) \text {. }
$$

Quantenmechanik war überaus erfolgreich, um z.B. die Stabilität von Atomen zu beschreiben. Im klassischen Bild umkreist im Wasserstoffatom ein Elektron auf einer gekrümmten Bahn den Atomkern, müsste also ständig als beschleunigtes geladenes Teilchen Energie abstrahlen und deshalb sich ständig dem Kern nähern, das Atom also kollabieren. Quantenmechanisch folgt aus der Unschärferelation, dass man Energie braucht, um das Elektron auf einen kleinen Raum wie eine kleine Umgebung des Kerns einzuengen. Dies hält den Aufenthaltsbereich des Elektrons gross im Vergleich zum Atomkern.

Quantenfeldtheorie versucht, die oben beschriebenen Konzepte von Teilchensystemen (endlich viele Freiheitsgrade) auf Felder (unendlich viele Freiheitsgrade) auszudehnen. Da in der Quantenmechanik Teilchen durch Felder beschrieben werden, redet man deshalb manchmal von zweiter Quantisierung. Notwendig wird sie, wenn man Relativitätstheorie und Quantenmechanik vereinigen will. Sie dient aber heute auch als die Sprache, in der die elementaren Kräfte (oder zumindest deren bestimmende Effekte) der Teilchenphysik beschrieben werden. Eine hervorragende Rolle spielen dabei die Eichfelder als Träger der Teilchen für die Wechselwirkungen zwischen Elementarteilchen.

Was eine Quantenfeldtheorie mathematisch sein soll, darüber besteht heute im wesentlichen Übereinstimmung. Sie wird durch ein System von Postulaten (Axiomen) beschrieben. Für Felder über der Raum-Zeit mit Minkowski-Metrik sind diese um 1960 von A.S. Wightman formuliert worden [2].

Wir wollen diese Axiome nicht alle auflisten und vermerken nur:

- Wir benötigen wieder einen Hilbertraum $\mathscr{H}$, der die Quantenzustände beschreibt.

- Auf $\mathscr{H}$ operiert die Poincarégruppe, d.h. die Isometriegruppe des Minkowskiraums, durch unitäre Transformationen. Insbesondere haben wir damit eine Zeitentwicklung und somit einen Hamiltonoperator.

- Observable sind nun operatorwertige Distributionen: Jeder samt aller ihrer Ableitungen bei unendlich schnell abklingender Funktion $f$ auf $\mathbb{R}^{d}$ wird ein dicht definierter selbstadjungierter Operator $\varphi(f)$ zugeordnet.

- Weiter, und das macht oft Schwierigkeiten, wird die Existenz eines eindeutig bestimmten Vakuums mit gewissen Eigenschaften gefordert.

Um die anstehenden Probleme einzuordnen, sollte man festhalten, dass selbst für relativ harmlose Felder wie so genannte skalare Felder, die Existenz einer Quantenfeldtheorie mit relativ allgemeinen Wechselwirkungen nur für 2-dimensionale Raum-Zeiten (also für Raumdimension 1) mathematisch exakt nachgewiesen ist. Für 4-dimensionale Raum-Zeiten gibt es, soweit ich weiss, keine Quantenfeldtheorie im obigen Sinne mit physikalisch relevanten Wechselwirkungen. 
Für Eichfeldtheorien zu einfachen Liegruppen $G$ wie $S U(n), n \geq 2$, kommt abgesehen von der Berücksichtigung der Eichtransformationen, oder gerade wegen deren Berücksichtigung, die enorme Schwierigkeit hinzu, eine Massenlücke nachzuweisen. Wir haben schon angedeutet, dass klassische Eichfelder wegen der geforderten Invarianz unter der Operation der Eichtransformationen masselos sind. Kräfte, die durch Austausch von masselosen Teilchen beschrieben werden, haben unendliche Reichweite. Deswegen möchte man Teilchen, die Wechselwirkungen mit kurzer Reichweite beschreiben, wie z.B. die Wechselwirkungen zwischen Hadronen (starke Wechselwirkung), Masse zuordnen können. Daher der Wunsch nach einer Massenlücke.

Was soll man sich mathematisch darunter vorstellen? Erinnern Sie sich an Einsteins berühmte Gleichung $E=m c^{2}$, die besagt, dass Masse und Energie zwei Seiten derselben Münze sind. Eigenwerte bzw. Spektralwerte des Energie- oder Hamiltonoperators beschreiben dann die möglichen Massen. Das Vakuum hat den Eigenwert 0, da es unter Zeittranslationen invariant ist. Die Theorie hat eine Massenlücke $m$, falls das Spektrum des Energieoperators zwischen 0 und $m$ keine Werte enthält. Die Existenz einer Massenlücke müssten Sie nachweisen, und das für Quanteneichfeldtheorien über dem $\mathbb{R}^{4}$ und zwar für jede einfache kompakte Liegruppe.

\section{Literatur}

[1] Jaffe, A.; Witten, E.: Yang-Mills Existence and Mass Gap. http://www.claymath.org/prizeproblems/yangmills.htm

[2] Streater, R.F.; Wightman, A.S.: PCT, Spin and Statistics, and All That. Benjamin, New York, 1964.

Elmar Vogt

Institut für Mathematik

Freie Universität Berlin

Arnimallee 2-6

D-14195 Berlin

e-mail: vogt@math.fu-berlin.de 